\title{
Development of Novel Diastereoselective Alkenylation of Enolates Using Alkenylselenonium Salts
}

\author{
Shin-ichi Watanabe, Takahiro Ikeda, Tadashi Kataoka,* Genzoh Tanabe, and Osamu Muraoka
}

Gifu Pharmaceutical University, 6-1 Mitahora-higashi 5-chome, Gifu 502-8585, Japan

\section{Supporting Information}

General. Melting points were obtained with a Yanagimoto micro-melting-point apparatus and are uncorrected. IR spectra of solids $(\mathrm{KBr})$ and liquids $(\mathrm{NaCl})$ were recorded on a JASCO FT/IR-230 spectrophotometer. ${ }^{1}$ HNMR spectra were recorded on a JEOL GX-270 (270 MHz) or a JEOL EX-400 (400 MHz) spectrometer with tetramethylsilane as an internal standard. ${ }^{13} \mathrm{CNMR}$ spectra were obtained on a JEOL EX-400 $(100 \mathrm{MHz})$ spectrometer with chloroform as an internal standard. The $J$ values are given in Hz. Mass spectra (MS and HRMS) were recorded on a JEOL JMS-SX102A spectrometer with an electron impact (EI, $70 \mathrm{eV}$ ) or fast atom bombardment (FAB, glycerol or 3-nitrobenzylalcohol) techniques. Elemental analyses of new compounds were performed by a Yanaco CHN Corder MT-5. All chromatographic isolations were accomplished with BW127ZH (Fuji Silysia) for column chromatography or with Kieselgel $60 \mathrm{PF}_{254}$ containing gypsum (Merck) for preparative TLC. Organic solvents used were dried by standard methods. The recycling preparative HPLC was performed by LC-918 liquid chromatography (Japan Analytical Industry Co., Ltd.) equipped with JAIGEL-1H and $-2 \mathrm{H}$ columns (polystyrene gel). Compounds $5 \mathrm{~g},{ }^{1} 5 \mathrm{~h}^{2}, 5 \mathrm{i}^{3}$ and $5 \mathrm{j}^{4}$ were prepared according to a literature method.

General procedure for the alkenylation reaction of achiral enolates with alkenylselenonium salt 1a: In a typical experiment, LHMDS (1 M in THF, $0.27 \mathrm{mmol})$ was dissolved in anhydrous THF (2 $\mathrm{mL})$ and the corresponding carbonyl compound $(0.24 \mathrm{mmol})$ was added at room temperature. After stirring for $30 \mathrm{~min}$ at same temperature, the reaction mixture was cooled to $-78^{\circ} \mathrm{C}$ followed by the addition of a THF solution $(2 \mathrm{~mL})$ of alkenylselenonium salt $1 \mathrm{a}(0.20 \mathrm{mmol})$ and the solution was stirred at same temperature for $2 \mathrm{~h}$. The mixture was worked with water and extracted with ethyl acetate. The organic layers were combined, washed with brine, dried over $\mathrm{MgSO}_{4}$, filtered, and concentrated. The crude products were separated by preparative TLC (silica gel, hexane/ethyl acetate mixtures) to give the corresponding alkenylation product.

(Z)-4-Benzenesulfonyl-2-methyl-1,4-diphenyl-3-buten-1-one (3a):

colorless needls (AcOEt-hexane), mp 115-116 ${ }^{\circ}$; IR $(\mathrm{NaCl}) / \mathrm{cm}^{-1} 1684,1446,1306,1151 ;{ }^{1} \mathrm{H}$ NMR $\left(\mathrm{CDCl}_{3}\right) \delta$ : $1.48\left(3 \mathrm{H}, \mathrm{d}, J=6.8 \mathrm{~Hz}, \mathrm{CHCH}_{3}\right), 5.90\left(1 \mathrm{H}, \mathrm{dq}, J=10.0\right.$ and $\left.6.8 \mathrm{~Hz}, \mathrm{CHCH}_{3}\right), 6.49(1 \mathrm{H}, \mathrm{d}, J=10.0 \mathrm{~Hz}$, $\mathrm{CH}=\mathrm{C}), 7.16-7.29(5 \mathrm{H}, \mathrm{m}, \mathrm{ArH}), 7.33(2 \mathrm{H}, \mathrm{t}, J=7.3 \mathrm{~Hz}, \operatorname{Ar} H), 7.47(1 \mathrm{H}, \mathrm{t}, J=7.3 \mathrm{~Hz}, \operatorname{Ar} H), 7.53(2 \mathrm{H}, \mathrm{t}, J=$ $7.3 \mathrm{~Hz}, \operatorname{Ar} H), 7.57(2 \mathrm{H}, \mathrm{d}, J=8.3 \mathrm{~Hz}, \operatorname{Ar} H), 7.62(1 \mathrm{H}, \mathrm{t}, J=7.3 \mathrm{~Hz}, \operatorname{Ar} H), 8.12(2 \mathrm{H}, \mathrm{d}, J=8.3 \mathrm{~Hz}, \operatorname{Ar} H) ;{ }^{13} \mathrm{C}$ $\operatorname{NMR}\left(\mathrm{CDCl}_{3}\right) \delta: 19.2,40.0,127.9,128.0,128.70,128.74,128.8,130.1,133.3,133.5,135.0,135.8,139.9$, 141.9, 144.6, 200.8; MS (EI) $\mathrm{m} / z 376\left(\mathrm{M}^{+}\right)$; Anal. Calcd for $\mathrm{C}_{23} \mathrm{H}_{20} \mathrm{O}_{3} \mathrm{~S}$ : C, 73.38; H, 5.35. Found: C, 73.34; $\mathrm{H}$, 5.38 .

(Z)-4-Benzenesulfonyl-2-benzyl-1,4-diphenyl-3-buten-1-one (3b):

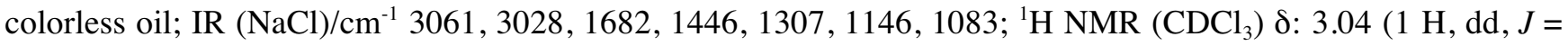
13.7 and $7.3 \mathrm{~Hz}, \mathrm{CHHPh}), 3.31(1 \mathrm{H}, \mathrm{dd}, J=13.7$ and $4.9 \mathrm{~Hz}, \mathrm{CHHPh}), 6.30-6.40\left(2 \mathrm{H}, \mathrm{m}, \mathrm{CHCH}_{2} \mathrm{Ph}\right.$ and $\mathrm{CH}=\mathrm{C}), 7.02(2 \mathrm{H}, \mathrm{d}, J=8.1 \mathrm{~Hz}, \operatorname{Ar} H), 7.13-7.34(12 \mathrm{H}, \mathrm{m}, \mathrm{ArH}), 7.40(1 \mathrm{H}, \mathrm{t}, J=6.8 \mathrm{~Hz}, \operatorname{Ar} H), 7.51(2 \mathrm{H}, \mathrm{t}, J$ $=7.6 \mathrm{~Hz}, \mathrm{Ar} H), 7.60(1 \mathrm{H}, \mathrm{t}, J=7.6 \mathrm{~Hz}, \mathrm{Ar} H), 8.14(2 \mathrm{H}, \mathrm{d}, J=7.6 \mathrm{~Hz}, \mathrm{Ar} H) ;{ }^{13} \mathrm{C} \mathrm{NMR}\left(\mathrm{CDCl}_{3}\right) \delta: 40.1,46.9$, $126.7,127.9,128.0,128.5,128.6,128.7,128.79,128.83,129.5,130.0,133.1,133.6,135.0,136.3,137.9,139.3$, 142.9, 143.0, 200.1; HRMS (EI) calcd for $\mathrm{C}_{29} \mathrm{H}_{24} \mathrm{O}_{3} \mathrm{~S}\left(\mathrm{M}^{+}\right)$452.1446, found 452.1442.

(1) Gage, J. R.; Evans, D. A. Org. Synth. 1990, 68, 83.

(2) Tao, T.; Parry, R. J. Org. Lett. 2001, 3, 3045.

(3) Evans, D. A.; Britton, T. C.; Dellaria, J. F., Jr. Tetrahedron 1988, 44, 5525.

(4) Edmonds, M. K.; Abell, A. D. J. Org. Chem. 2001, 66, 3747. 
(Z)-4-Benzenesulfonyl-1,4-diphenyl-2-propyl-3-buten-1-one (3c):

colorless oil; IR $(\mathrm{NaCl}) / \mathrm{cm}^{-1} 2959,2931,1682,1446,1306,1150,1085 ;{ }^{1} \mathrm{H} \mathrm{NMR}\left(\mathrm{CDCl}_{3}\right) \delta: 0.96(3 \mathrm{H}, \mathrm{t}, J=$ $\left.7.3 \mathrm{~Hz}, \mathrm{CH}_{2} \mathrm{CH}_{3}\right), 1.42-1.51\left(2 \mathrm{H}, \mathrm{m}, \mathrm{CH}_{2} \mathrm{CH}_{3}\right), 1.62-1.70\left(1 \mathrm{H}, \mathrm{m}, \mathrm{CH} \mathrm{HCH}_{2} \mathrm{CH}_{3}\right), 1.92-1.98(1 \mathrm{H}, \mathrm{m}$, $\left.\mathrm{CHHCH}_{2} \mathrm{CH}_{3}\right), 5.95\left(1 \mathrm{H}\right.$, ddd, $J=10.3$ and 7.8 and $\left.5.9 \mathrm{~Hz}, \mathrm{CHCH}_{2}\right), 6.41(1 \mathrm{H}, \mathrm{d}, J=10.3 \mathrm{~Hz}, \mathrm{CH}=\mathrm{C}), 7.15-$ $7.36(7 \mathrm{H}, \mathrm{m}, \operatorname{Ar} H), 7.44(1 \mathrm{H}, \mathrm{t}, J=7.3 \mathrm{~Hz}, \operatorname{Ar} H), 7.53(2 \mathrm{H}, \mathrm{d}, J=7.3 \mathrm{~Hz}, \operatorname{Ar} H), 7.54(2 \mathrm{H}, \mathrm{t}, J=7.3 \mathrm{~Hz}, \operatorname{Ar} H)$, $7.63(1 \mathrm{H}, \mathrm{t}, J=7.3 \mathrm{~Hz}, \mathrm{ArH}), 8.15(2 \mathrm{H}, \mathrm{d}, J=7.3 \mathrm{~Hz}, \mathrm{ArH}) ;{ }^{13} \mathrm{C} \mathrm{NMR}\left(\mathrm{CDCl}_{3}\right)$ 8: 14.0, 20.6, 36.3, 45.1, 127.9, 128.0, 128.6, 128.7, 128.8, 130.1, 133.2, 133.5, 135.1, 136.5, 139.8, 142.7, 144.4, 200.9; HRMS (EI) calcd for $\mathrm{C}_{25} \mathrm{H}_{24} \mathrm{O}_{3} \mathrm{~S}\left(\mathrm{M}^{+}\right)$404.1446, found 404.1435 .

(Z)-2-(2-Benzenesulfonyl-2-phenylethenyl)cyclohexanone (3d):

colorless oil; IR $(\mathrm{NaCl}) / \mathrm{cm}^{-1} 2939,1709,1446,1307,1149,1084,768,731,689,586 ;{ }^{1} \mathrm{H} \mathrm{NMR}\left(\mathrm{CDCl}_{3}\right) \delta: 1.60-$ $1.80(2 \mathrm{H}, \mathrm{m}, \mathrm{CHH}), 1.84-2.02(2 \mathrm{H}, \mathrm{m}, \mathrm{CHH}), 2.15-2.23(1 \mathrm{H}, \mathrm{m}, \mathrm{CHH}), 2.38-2.46(1 \mathrm{H}, \mathrm{m}, \mathrm{CHH}), 2.46-2.53(2$ $\mathrm{H}, \mathrm{m}, \mathrm{CHH}), 4.78(1 \mathrm{H}$, ddd, $J=12.3$ and 9.3 and $5.4 \mathrm{~Hz}, \mathrm{C}(\mathrm{O}) \mathrm{CHCH} 2), 6.38(1 \mathrm{H}, \mathrm{d}, J=9.3 \mathrm{~Hz}, \mathrm{CH}=\mathrm{C}), 7.16-$ $7.27(5 \mathrm{H}, \mathrm{m}, \mathrm{ArH}), 7.35(2 \mathrm{H}, \mathrm{t}, J=7.8 \mathrm{~Hz}, \mathrm{ArH}), 7.48(1 \mathrm{H}, \mathrm{t}, J=7.8 \mathrm{~Hz}, \mathrm{ArH}), 7.59(2 \mathrm{H}, \mathrm{d}, J=7.8 \mathrm{~Hz}, \mathrm{ArH})$; ${ }^{13} \mathrm{C} \mathrm{NMR}\left(\mathrm{CDCl}_{3}\right) \delta: 24.8,27.8,35.4,42.1,49.8,127.9,128.0,128.6,130.1,133.2,135.1,139.9,142.3,142.6$, 209.5; MS (FAB) m/z $341(\mathrm{M}+\mathrm{H})^{+}$; Anal. Calcd for $\mathrm{C}_{20} \mathrm{H}_{20} \mathrm{O}_{3} \mathrm{~S}$ : C, 70.56; H, 5.92. Found: C, 70.29; H, 6.09.

(Z)-4-(2-Benzenesulfonyl-2-phenylethenyl)isochroman-3-one (3e):

white crystal (AcOEt-hexane), mp $171^{\circ} \mathrm{C}$; IR $(\mathrm{NaCl}) / \mathrm{cm}^{-1} 1730,1308,1147,1084 ;{ }^{1} \mathrm{H} \mathrm{NMR}\left(\mathrm{CDCl}_{3}\right) \delta: 5.13(2$ $\left.\mathrm{H}, \mathrm{s}, \mathrm{CH}_{2} \mathrm{O}\right), 6.47(1 \mathrm{H}, \mathrm{d}, J=10.7 \mathrm{~Hz}, \mathrm{CHCH}=\mathrm{C}), 6.91(1 \mathrm{H}, \mathrm{d}, J=10.7 \mathrm{~Hz}, \mathrm{CHCH}=\mathrm{C}), 7.19(1 \mathrm{H}, \mathrm{d}, J=6.8$ $\mathrm{Hz}, \operatorname{Ar} H), 7.26-7.50(10 \mathrm{H}, \mathrm{m}, \operatorname{Ar} H), 7.59(1 \mathrm{H}, \mathrm{t}, J=7.3 \mathrm{~Hz}, \operatorname{Ar} H), 7.72(2 \mathrm{H}, \mathrm{d}, J=7.3 \mathrm{~Hz}, \operatorname{Ar} H) ;{ }^{13} \mathrm{C}$ NMR

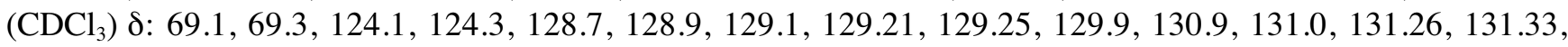
132.2, 133.8, 137.6, 164.9; MS (FAB) $\mathrm{m} / z 391(\mathrm{M}+\mathrm{H})^{+}$; Anal. Calcd for $\mathrm{C}_{23} \mathrm{H}_{18} \mathrm{O}_{4} \mathrm{~S}: \mathrm{C}, 70.75 ; \mathrm{H}, 4.65$. Found: $\mathrm{C}$, $70.50 ; \mathrm{H}, 4.72$.

(Z)-4-Benzenesulfonyl-2,2-dimethyl-1,4-diphenyl-3-buten-1-one (3f):

pale purple needls (AcOEt-hexane), mp $188-189^{\circ} \mathrm{C}$; IR $(\mathrm{NaCl}) / \mathrm{cm}^{-1} 1683,1446,1306,1148 ;{ }^{1} \mathrm{H} \mathrm{NMR}\left(\mathrm{CDCl}_{3}\right)$

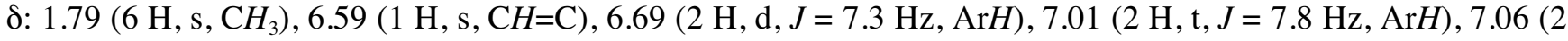
$\mathrm{H}, \mathrm{d}, J=7.3 \mathrm{~Hz}, \operatorname{Ar} H), 7.15(2 \mathrm{H}, \mathrm{t}, J=7.3 \mathrm{~Hz}, \operatorname{Ar} H), 7.22(1 \mathrm{H}, \mathrm{t}, J=7.3 \mathrm{~Hz}, \operatorname{Ar} H), 7.28(1 \mathrm{H}, \mathrm{t}, J=7.3 \mathrm{~Hz}$, $\operatorname{Ar} H), 7.53(2 \mathrm{H}, \mathrm{t}, J=7.3 \mathrm{~Hz}, \operatorname{Ar} H), 7.61(1 \mathrm{H}, \mathrm{t}, J=7.3 \mathrm{~Hz}, \operatorname{Ar} H), 8.05(2 \mathrm{H}, \mathrm{d}, J=7.3 \mathrm{~Hz}, \operatorname{Ar} H) ;{ }^{13} \mathrm{C}$ NMR

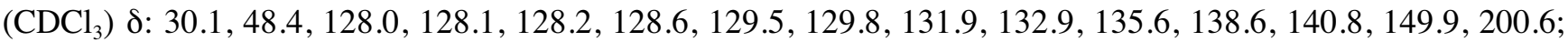
MS (EI) $\mathrm{m} / z 390\left(\mathrm{M}^{+}\right)$; Anal. Calcd for $\mathrm{C}_{24} \mathrm{H}_{22} \mathrm{O}_{3} \mathrm{~S}: \mathrm{C}$, 73.82; H, 5.68. Found: C, 73.65; H, 5.80.

(Z)-2-Benzenesulfonyl-2-phenylethenyl 2,2-dimethyl-1-phenylethenyl ether (4):

yellow oil; IR $(\mathrm{NaCl}) / \mathrm{cm}^{-1}$ 3059, 2916, 1621, 1445, 1307, 1194, 1144, 1083, 1017, 766, 724, 699, 689, 600, 559; ${ }^{1} \mathrm{H} \mathrm{NMR}\left(\mathrm{CDCl}_{3}\right) \delta: 1.75\left(3 \mathrm{H}, \mathrm{s}, E-\mathrm{CH}_{3}\right), 1.79\left(3 \mathrm{H}, \mathrm{s}, Z-\mathrm{CH}_{3}\right), 6.47(1 \mathrm{H}, \mathrm{s}, \mathrm{CH}=\mathrm{C}), 7.08(2 \mathrm{H}, \mathrm{d}, J=7.6 \mathrm{~Hz}$, $\operatorname{Ar} H)$, 7.15-7.30 (8 H, m, ArH $), 7.50(2 \mathrm{H}, \mathrm{t}, J=7.3 \mathrm{~Hz}, \operatorname{Ar} H), 7.59(1 \mathrm{H}, \mathrm{t}, J=7.3 \mathrm{~Hz}, \operatorname{Ar} H), 7.97(2 \mathrm{H}, \mathrm{d}, J=$ $7.3 \mathrm{~Hz}, \mathrm{Ar} H) ;{ }^{13} \mathrm{C} \mathrm{NMR}\left(\mathrm{CDCl}_{3}\right) \delta: 18.1,19.9,120.1,122.6,127.7,128.1,128.26,128.32,128.5,129.0,130.8$, 131.5, 132.7, 133.9, 142.7, 146.5, 152.8; MS (EI) $\mathrm{m} / z 390(\mathrm{M})^{+}$; HRMS calcd for $\mathrm{C}_{24} \mathrm{H}_{22} \mathrm{O}_{3} \mathrm{~S}\left(\mathrm{M}^{+}\right) 390.1289$, found 390.1281 .

General procedure for the alkenylation reaction of chiral enolates with alkenylselenonium salts 1: LHMDS (1 $\mathrm{M}$ in THF, $0.23 \mathrm{mmol}$ ) was added to a THF solution $(1.6 \mathrm{~mL})$ of the corresponding oxazolidinone derivative $(0.2 \mathrm{mmol})$ at $-78^{\circ} \mathrm{C}$. After stirring for $1 \mathrm{~h}$ at same temperature, the reaction mixture was added to a THF solution $(2.4 \mathrm{~mL})$ of alkenylselenonium salt $1(0.20 \mathrm{mmol})$ and the solution was stirred at $-78^{\circ} \mathrm{C}$ for $2 \mathrm{~h}$. The mixture was worked with water and extracted with ethyl acetate. The organic layers were combined, washed with brine, dried over $\mathrm{MgSO}_{4}$, filtered, and concentrated. The crude products were separated by preparative TLC (silica gel, hexane/ethyl acetate mixtures) or recycling preparative $\operatorname{HPLC}\left(\mathrm{CHCl}_{3}\right)$ to give the corresponding alkenylation product. Diastereoselectivities were determined by ${ }^{1} \mathrm{H}-\mathrm{NMR}$ measurement.

(4S)-3-[(Z)-(2R)-4-Benzenesulfonyl-2-methyl-1-oxo-4-phenyl]-4-phenylmethyl-2-oxazolidinone (3g) (ma jor 
product):

white powder, $\mathrm{mp} 51-52^{\circ} \mathrm{C}$; IR $(\mathrm{NaCl}) / \mathrm{cm}^{-1} 3061,3029,1790,1697,1446,1387,1361,1308,1211,1146,975$,

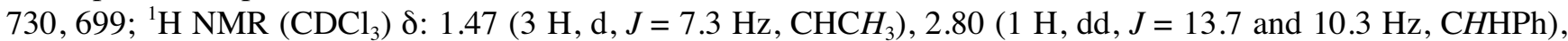
$3.44(1 \mathrm{H}, \mathrm{dd}, J=13.7$ and $3.4 \mathrm{~Hz}, \mathrm{CH} H \mathrm{Ph}), 4.19-4.26\left(2 \mathrm{H}, \mathrm{m}, \mathrm{NCHCH}_{2}\right), 4.70(1 \mathrm{H}$, ddd, $J=13.7$ and 6.8 and $\left.3.4 \mathrm{~Hz}, \mathrm{NCHCH}_{2}\right), 5.72\left(1 \mathrm{H}, \mathrm{dq}, J=8.8\right.$ and $\left.7.3 \mathrm{~Hz}, \mathrm{CHCH}_{3}\right), 6.35(1 \mathrm{H}, \mathrm{d}, J=8.8 \mathrm{~Hz}, \mathrm{CH}=\mathrm{C}), 7.24-7.34(10$ $\mathrm{H}, \mathrm{m}, \mathrm{Ar} H), 7.39(2 \mathrm{H}, \mathrm{t}, J=7.3 \mathrm{~Hz}, \operatorname{Ar} H), 7.51(1 \mathrm{H}, \mathrm{t}, J=7.3 \mathrm{~Hz}, \operatorname{Ar} H), 7.70(2 \mathrm{H}, \mathrm{d}, J=7.3 \mathrm{~Hz}, \operatorname{Ar} H) ;{ }^{13} \mathrm{C}$ NMR $\left(\mathrm{CDCl}_{3}\right) \delta: 19.3,37.6,38.0,55.6,66.1,127.2,128.0,128.1,128.77,128.81,128.9,129.5,130.1,133.3$, 134.9, 135.6, 140.2, 143.2, 143.4, 152.7, 174.5; MS (EI) $\mathrm{m} / z \quad 475(\mathrm{M})^{+}$; Anal. Calcd for $\mathrm{C}_{27} \mathrm{H}_{25} \mathrm{NO}_{5} \mathrm{~S}: \mathrm{C}, 68.19$; H, 5.30; N, 2.95. Found: C, 68.05; H, 5.38; N, 2.90 .

(4S)-3-[(Z)-(2S)-4-Benzenesulfonyl-2-methyl-1-oxo-4-phenyl]-4-phenylmethyl-2-oxazolidinone (3g) (min or product):

white powder, $\mathrm{mp} 53-55^{\circ} \mathrm{C}$; IR $(\mathrm{NaCl}) / \mathrm{cm}-1$ 3061, 3029, 2925, 1789, 1695, 1446, 1386, 1360, 1308, 1211, 1145 , 976, 761, 731, 699; ${ }^{1} \mathrm{H}$ NMR $\left(\mathrm{CDCl}_{3}\right) \delta: 1.49(3 \mathrm{H}, \mathrm{d}, J=7.3 \mathrm{~Hz}, \mathrm{CHCH3}), 2.86(1 \mathrm{H}, \mathrm{dd}, J=13.4 \mathrm{and} 9.3 \mathrm{~Hz}$, $\mathrm{CHHPh}), 3.26(1 \mathrm{H}, \mathrm{dd}, J=13.4$ and $3.4 \mathrm{~Hz}, \mathrm{CH} H \mathrm{Ph}), 4.22(1 \mathrm{H}, \mathrm{dd}, J=8.5$ and $2.7 \mathrm{~Hz}, \mathrm{NCHCHH}), 4.32(1 \mathrm{H}$, $\mathrm{t}, J=8.5 \mathrm{~Hz}, \mathrm{NCHCHH}), 4.76(1 \mathrm{H}$, dddd, $J=9.3$ and 8.5 and 3.4 and $2.7 \mathrm{~Hz}, \mathrm{NCHCH} 2), 5.57(1 \mathrm{H}, \mathrm{dq}, J=8.6$ and $7.3 \mathrm{~Hz}, \mathrm{CHCH} 3), 6.35(1 \mathrm{H}, \mathrm{d}, J=8.6 \mathrm{~Hz}, \mathrm{CH}=\mathrm{C}), 7.22-7.37(10 \mathrm{H}, \mathrm{m}, \mathrm{ArH}), 7.40(2 \mathrm{H}, \mathrm{t}, J=7.6 \mathrm{~Hz}, \mathrm{ArH})$, $7.52(1 \mathrm{H}, \mathrm{t}, J=7.6 \mathrm{~Hz}, \mathrm{Ar} H), 7.66(2 \mathrm{H}, \mathrm{d}, J=7.6 \mathrm{~Hz}, \mathrm{Ar} H) ;{ }^{13} \mathrm{C} \mathrm{NMR}\left(\mathrm{CDCl}_{3}\right)$ 8: 19.3, 37.8, 38.1, 55.1, 66.4, $127.4,127.99,128.02,128.81,128.85,128.9,129.5,130.1,133.3,134.8,135.1,140.2,143.2,143.6,153.0$, 174.5; MS (EI) $\mathrm{m} / z 475(\mathrm{M})^{+}$; Anal. Calcd for $\mathrm{C}_{27} \mathrm{H}_{25} \mathrm{NO}_{5} \mathrm{~S}: \mathrm{C}, 68.19 ; \mathrm{H}, 5.30 ; \mathrm{N}, 2.95$. Found: C, 67.95; H, 5.22; N, 2.79 .

(4S)-3-[(Z)-(2R)-4-Benzenesulfonyl-2-ethyl-1-oxo-4-phenyl]-4-phenylmethyl-2-oxazolidinone（ $3 \mathrm{~h}$ ) (m a j o r product):

pale yellow viscous oil; IR $(\mathrm{NaCl}) / \mathrm{cm}^{-1} 2924,1780,1693,1446,1387,1308,1210,1148,918,761,730,700 ;{ }^{1} \mathrm{H}$ $\operatorname{NMR}\left(\mathrm{CDCl}_{3}\right) \delta: 0.97\left(3 \mathrm{H}, \mathrm{t}, J=7.3 \mathrm{~Hz}, \mathrm{CHCH}_{2} \mathrm{CH}_{3}\right), 1.73(1 \mathrm{H}$, ddq, $J=13.2$ and 8.8 and $7.3 \mathrm{~Hz}$, $\left.\mathrm{CHCHHCH}_{3}\right), 1.98\left(1 \mathrm{H}\right.$, ddq, $J=13.2$ and 7.3 and $\left.4.4 \mathrm{~Hz}, \mathrm{CHCHHCH}_{3}\right), 2.80(1 \mathrm{H}$, dd, $J=13.7$ and $10.3 \mathrm{~Hz}$, $\mathrm{C} H \mathrm{HPh}), 3.45(1 \mathrm{H}, \mathrm{dd}, J=13.7$ and $3.4 \mathrm{~Hz}, \mathrm{CH} H \mathrm{Ph}), 4.19-4.24\left(2 \mathrm{H}, \mathrm{m}, \mathrm{NCHCH}_{2}\right), 4.67-4.72(1 \mathrm{H}, \mathrm{m}$, $\left.\mathrm{NCHCH}_{2}\right), 5.63\left(1 \mathrm{H}, \mathrm{dt}, J=8.8\right.$ and $\left.4.4 \mathrm{~Hz}, \mathrm{CHCH}_{2} \mathrm{CH}_{3}\right), 6.32(1 \mathrm{H}, \mathrm{d}, J=8.8 \mathrm{~Hz}, \mathrm{CH}=\mathrm{C}), 7.25-7.35(10 \mathrm{H}, \mathrm{m}$, $\mathrm{Ar} H), 7.40(2 \mathrm{H}, \mathrm{t}, J=7.8 \mathrm{~Hz}, \operatorname{Ar} H), 7.51(1 \mathrm{H}, \mathrm{t}, J=7.8 \mathrm{~Hz}, \mathrm{Ar} H), 7.72(2 \mathrm{H}, \mathrm{d}, J=7.8 \mathrm{~Hz}, \mathrm{Ar} H) ;{ }^{13} \mathrm{C}$ NMR

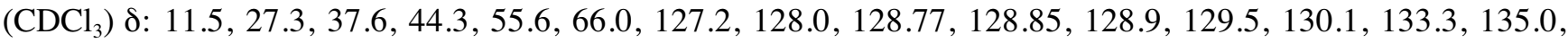
135.6, 140.4, 143.0, 144.7, 152.9, 173.7; HRMS calcd for $\mathrm{C}_{28} \mathrm{H}_{27} \mathrm{NO}_{5} \mathrm{~S}\left(\mathrm{M}^{+}\right) 489.1609$, found 489.1607 .

(4S)-3-[(Z)-(2R)-4-Benzenesulfonyl-2-ethyl-1-oxo-4-phenyl]-4-phenylmethyl-2-oxazolidinone ( $3 h$ ) (m i n o r product):

white powder, $\mathrm{mp} 44-47^{\circ} \mathrm{C}$; IR $(\mathrm{NaCl}) / \mathrm{cm}^{-1} 2925,1780,1694,1446,1387,1308,1211,1146,918,761,730,699$ ; ${ }^{1} \mathrm{H}$ NMR $\left(\mathrm{CDCl}_{3}\right) \delta: 0.99\left(3 \mathrm{H}, \mathrm{t}, J=7.3 \mathrm{~Hz}, \mathrm{CHCH}_{2} \mathrm{CH}_{3}\right), 1.75(1 \mathrm{H}, \mathrm{ddq}, J=13.2$ and 8.8 and $7.3 \mathrm{~Hz}$, $\left.\mathrm{CHCHHCH}_{3}\right), 2.04\left(1 \mathrm{H}\right.$, ddq, $J=13.2$ and 7.3 and $\left.4.4 \mathrm{~Hz}, \mathrm{CHCHHCH}_{3}\right), 2.81(1 \mathrm{H}, \mathrm{dd}, J=13.7$ and $9.8 \mathrm{~Hz}$, $\mathrm{C} H \mathrm{HPh}), 3.29(1 \mathrm{H}, \mathrm{dd}, J=13.7$ and $3.4 \mathrm{~Hz}, \mathrm{CH} H \mathrm{Ph}), 4.21(1 \mathrm{H}, \mathrm{dd}, J=8.8$ and $2.4 \mathrm{~Hz}, \mathrm{NCHCHH}), 4.31(1 \mathrm{H}$, $\mathrm{t}, J=8.8 \mathrm{~Hz}, \mathrm{NCHCHH}), 4.75\left(1 \mathrm{H}\right.$, dddd, $J=9.8$ and 8.8 and 3.4 and $\left.2.4 \mathrm{~Hz}, \mathrm{NCHCH}_{2}\right), 5.46(1 \mathrm{H}, \mathrm{dt}, J=8.8$ and $\left.4.4 \mathrm{~Hz}, \mathrm{CHCH}_{2} \mathrm{CH}_{3}\right), 6.32(1 \mathrm{H}, \mathrm{d}, J=8.8 \mathrm{~Hz}, \mathrm{CH}=\mathrm{C}), 7.23-7.37(10 \mathrm{H}, \mathrm{m}, \mathrm{ArH}), 7.41(2 \mathrm{H}, \mathrm{t}, J=7.8 \mathrm{~Hz}$,

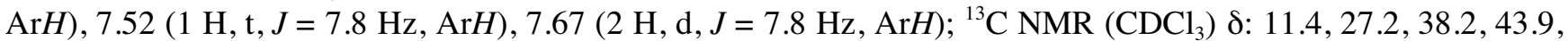
55.3, 66.3, 127.4, 127.99, 128.04, 128.8, 128.90, 128.94, 129.4, 130.1, 133.3, 135.0, 135.2, 140.4, 143.1, 144.7, 153.1, 173.6; HRMS calcd for $\mathrm{C}_{28} \mathrm{H}_{27} \mathrm{NO}_{5} \mathrm{~S}\left(\mathrm{M}^{+}\right) 489.1609$, found 489.1608 .

(4S)-3-[(Z)-(2R)-4-Benzenesulfonyl-2-isopropyl-1-oxo-4-phenyl]-4-phenylmethyl-2-oxazolidinone (major product):

colorless crystal $\left(\mathrm{CHCl}_{3}\right.$-ether), mp 126-127 ${ }^{\circ} \mathrm{C}$; IR $(\mathrm{NaCl}) / \mathrm{cm}^{-1} 2964,2929,1780,1692,1446,1386,1349,1307$, 1209, 1148, 761, 731, 700, 608, 578; ${ }^{1} \mathrm{H}$ NMR $\left(\mathrm{CDCl}_{3}\right) \delta: 0.96\left(3 \mathrm{H}, \mathrm{d}, J=6.8 \mathrm{~Hz}, \mathrm{CH}\left(\mathrm{CH}_{3}\right)\left(\mathrm{CH}_{3}\right)\right), 1.00(3 \mathrm{H}$, $\left.\mathrm{d}, J=6.8 \mathrm{~Hz}, \mathrm{CH}\left(\mathrm{CH}_{3}\right)\left(\mathrm{CH}_{3}\right)\right), 2.24-2.32\left(1 \mathrm{H}, \mathrm{m}, \mathrm{CH}\left(\mathrm{CH}_{3}\right)_{2}\right), 2.79(1 \mathrm{H}, \mathrm{dd}, J=13.7$ and $10.3 \mathrm{~Hz}, \mathrm{CHHPh})$, $3.47(1 \mathrm{H}, \mathrm{dd}, J=13.7$ and $3.4 \mathrm{~Hz}, \mathrm{CH} H \mathrm{Ph}), 4.20\left(2 \mathrm{H}, \mathrm{d}, J=4.9 \mathrm{~Hz}, \mathrm{NCHCH}{ }_{2}\right), 4.67-4.73\left(1 \mathrm{H}, \mathrm{m}, \mathrm{NCHCH}_{2}\right)$, $5.71\left(1 \mathrm{H}, \mathrm{dd}, J=9.3\right.$ and $\left.5.4 \mathrm{~Hz}, \mathrm{CHCH}\left(\mathrm{CH}_{3}\right)_{2}\right), 6.37(1 \mathrm{H}, \mathrm{d}, J=9.3 \mathrm{~Hz}, \mathrm{CH}=\mathrm{C}), 7.26-7.35(10 \mathrm{H}, \mathrm{m}, \mathrm{ArH})$, $7.41(2 \mathrm{H}, \mathrm{t}, J=7.8 \mathrm{~Hz}, \mathrm{Ar} H), 7.52(1 \mathrm{H}, \mathrm{t}, J=7.8 \mathrm{~Hz}, \mathrm{Ar} H), 7.73(2 \mathrm{H}, \mathrm{d}, J=7.8 \mathrm{~Hz}, \mathrm{Ar} H) ;{ }^{13} \mathrm{C} \mathrm{NMR}\left(\mathrm{CDCl}_{3}\right)$ 
ઠ: $17.9,20.5,31.6,37.5,47.9,55.7,66.0,127.2,128.0,128.8,128.87,128.92,129.5,130.2,133.3,135.3,135.7$, 140.6, 141.1, 145.4, 152.9, 173.2; MS (EI) $\mathrm{m} / z 503(\mathrm{M})^{+}$; Anal. Calcd for $\mathrm{C}_{29} \mathrm{H}_{29} \mathrm{NO}_{5} \mathrm{~S}$ : C, 69.16; H, 5.80; N, 2.78. Found: C, $68.98 ; \mathrm{H}, 5.82 ; \mathrm{N}, 2.58$.

(4S)-3-[(Z)-(2R)-4-Benzenesulfonyl-2-isopropyl-1-oxo-4-phenyl]-4-phenylmethyl-2-oxazolidinone (minor product):

white powder, $\mathrm{mp} 46-49^{\circ} \mathrm{C}$; IR $(\mathrm{NaCl}) / \mathrm{cm}^{-1} 2961,2925,1781,1692,1446,1387,1349,1307,1210,1148,761$,

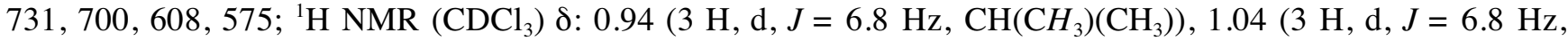
$\left.\mathrm{CH}\left(\mathrm{CH}_{3}\right)\left(\mathrm{CH}_{3}\right)\right), 2.30-2.39\left(1 \mathrm{H}, \mathrm{m}, \mathrm{CH}\left(\mathrm{CH}_{3}\right)_{2}\right), 2.75(1 \mathrm{H}, \mathrm{dd}, J=13.2$ and $10.3 \mathrm{~Hz}, \mathrm{CHHPh}), 3.36(1 \mathrm{H}, \mathrm{dd}, J=$ 13.2 and $2.9 \mathrm{~Hz}, \mathrm{CH} H \mathrm{Ph}), 4.19(1 \mathrm{H}, \mathrm{dd}, J=8.8$ and $2.4 \mathrm{~Hz}, \mathrm{NCHCHH}), 4.30(1 \mathrm{H}, \mathrm{t}, J=8.8 \mathrm{~Hz}, \mathrm{NCHCHH})$, 4.74-4.79 $\left.(1 \mathrm{H}, \mathrm{m}, \mathrm{NCHCH})_{2}\right), 5.50\left(1 \mathrm{H}, \mathrm{dd}, J=9.3\right.$ and $\left.5.9 \mathrm{~Hz}, \mathrm{CHCH}\left(\mathrm{CH}_{3}\right)_{2}\right), 6.36(1 \mathrm{H}, \mathrm{d}, J=9.3 \mathrm{~Hz}$, $\mathrm{C} H=\mathrm{C}), 7.25-7.37(10 \mathrm{H}, \mathrm{m}, \operatorname{Ar} H), 7.42(2 \mathrm{H}, \mathrm{t}, J=7.3 \mathrm{~Hz}, \operatorname{Ar} H), 7.53(1 \mathrm{H}, \mathrm{t}, J=7.3 \mathrm{~Hz}, \operatorname{Ar} H), 7.69(2 \mathrm{H}, \mathrm{d}, J$ $=7.3 \mathrm{~Hz}, \mathrm{ArH}) ;{ }^{13} \mathrm{C} \mathrm{NMR}\left(\mathrm{CDCl}_{3}\right) \delta: 18.0,20.4,31.8,38.4,47.5,55.5,66.3,127.3,128.0,128.1,128.8,128.9$, 129.0, 129.4, 130.2, 133.3, 135.2, 135.4, 140.5, 141.5, 145.2, 153.2, 173.1; HRMS calcd for $\mathrm{C}_{29} \mathrm{H}_{29} \mathrm{NO}_{5} \mathrm{~S}\left(\mathrm{M}^{+}\right)$ 503.1766 , found 503.1773.

(4S)-3-[(Z)-(2R)-4-Benzenesulfonyl-2-benzyl-1-oxo-4-phenyl]-4-phenylmethyl-2-oxazolidinone $\quad(3 \mathrm{j})(\mathrm{m}$ a j or product):

pale yellow viscous oil; IR $(\mathrm{NaCl}) / \mathrm{cm}^{-1} 2921,1779,1694,1446,1387,1307,1212,1148,922,732,699 ;{ }^{1} \mathrm{H}$ NMR $\left(\mathrm{CDCl}_{3}\right) \delta: 2.79(1 \mathrm{H}, \mathrm{dd}, J=13.2$ and $9.8 \mathrm{~Hz}, \mathrm{NCHCHHPh}), 3.04(1 \mathrm{H}, \mathrm{dd}, J=12.7$ and $8.8 \mathrm{~Hz}$, $\mathrm{C}(\mathrm{O}) \mathrm{CHCHHPh}), 3.25(1 \mathrm{H}, \mathrm{dd}, J=12.7$ and $6.4 \mathrm{~Hz}, \mathrm{C}(\mathrm{O}) \mathrm{CHCHHPh}), 3.41(1 \mathrm{H}, \mathrm{dd}, J=13.2$ and $2.4 \mathrm{~Hz}$, $\mathrm{NCHCH} H \mathrm{Ph}), 3.94(1 \mathrm{H}, \mathrm{t}, J=8.8 \mathrm{~Hz}, \mathrm{NCHCHHO}), 4.12(1 \mathrm{H}, \mathrm{dd}, J=8.8$ and $2.4 \mathrm{~Hz}, \mathrm{NCHCHHO}), 4.53(1 \mathrm{H}$, ddt, $J=9.8$ and 8.8 and $\left.2.4 \mathrm{~Hz}, \mathrm{NCHCH}_{2}\right), 6.15\left(1 \mathrm{H}, \mathrm{dt}, J=8.8\right.$ and $\left.6.4 \mathrm{~Hz}, \mathrm{C}(\mathrm{O}) \mathrm{CHCH}_{2} \mathrm{Ph}\right), 6.41(1 \mathrm{H}, \mathrm{d}, J=$ $8.8 \mathrm{~Hz}, \mathrm{CH}=\mathrm{C}), 7.09(2 \mathrm{H}, \mathrm{d}, J=7.8 \mathrm{~Hz}, \mathrm{ArH}), 7.20-7.36(15 \mathrm{H}, \mathrm{m}, \mathrm{Ar} H), 7.49(1 \mathrm{H}, \mathrm{t}, J=7.3 \mathrm{~Hz}, \operatorname{Ar} H), 7.53(2$ $\mathrm{H}, \mathrm{d}, J=7.3 \mathrm{~Hz}, \mathrm{ArH}) ;{ }^{13} \mathrm{C} \mathrm{NMR}\left(\mathrm{CDCl}_{3}\right) \delta: 37.6,40.6,44.8,55.7,66.1,126.9,127.2,127.9,128.0,128.4$, $128.7,128.8,129.0,129.3,129.5,130.2,133.2,134.9,135.5,137.4,139.9,142.2,144.1,152.8,173.3$; HRMS calcd for $\mathrm{C}_{33} \mathrm{H}_{29} \mathrm{NO}_{5} \mathrm{~S}\left(\mathrm{M}^{+}\right)$551.1766, found 551.1774 .

(4S)-3-[(Z)-(2R)-4-Benzenesulfonyl-2-benzyl-1-oxo-4-phenyl]-4-phenylmethyl-2-oxazolidinone (3j) (m in or product):

pale yellow viscous oil; IR $(\mathrm{NaCl}) / \mathrm{cm}^{-1} 2923,1779,1694,1446,1387,1307,1212,1147,923,760,731,700 ;{ }^{1} \mathrm{H}$ NMR $\left(\mathrm{CDCl}_{3}\right) \delta: 2.75(1 \mathrm{H}, \mathrm{dd}, J=13.2$ and $9.8 \mathrm{~Hz}, \mathrm{C}(\mathrm{O}) \mathrm{CHCHHPh}), 2.89(1 \mathrm{H}, \mathrm{dd}, J=13.2$ and $10.3 \mathrm{~Hz}$, $\mathrm{NCHCHHPh}), 3.24(1 \mathrm{H}, \mathrm{dd}, J=13.2$ and $3.4 \mathrm{~Hz}, \mathrm{NCHCHHPh}), 3.44(1 \mathrm{H}$, dd, $J=13.2$ and $4.4 \mathrm{~Hz}$, $\mathrm{C}(\mathrm{O}) \mathrm{CHCHHPh}$, $4.21(1 \mathrm{H}, \mathrm{dd}, J=8.8$ and $2.4 \mathrm{~Hz}, \mathrm{NCHCHHO}), 4.34(1 \mathrm{H}, \mathrm{t}, J=8.8 \mathrm{~Hz}, \mathrm{NCHCHHO}), 4.77$ (1 $\mathrm{H}$, dddd, $J=10.3$ and 8.8 and 3.4 and $\left.2.4 \mathrm{~Hz}, \mathrm{NCHCH}_{2}\right), 5.98\left(1 \mathrm{H}, \mathrm{dt}, J=9.8\right.$ and $\left.4.4 \mathrm{~Hz}, \mathrm{C}(\mathrm{O}) \mathrm{CHCH}_{2} \mathrm{Ph}\right), 6.33$ $(1 \mathrm{H}, \mathrm{d}, J=9.8 \mathrm{~Hz}, \mathrm{CH}=\mathrm{C}), 7.01(2 \mathrm{H}, \mathrm{d}, J=7.6 \mathrm{~Hz}, \operatorname{Ar} H), 7.18-7.38(17 \mathrm{H}, \mathrm{m}, \operatorname{Ar} H), 7.47(1 \mathrm{H}, \mathrm{t}, J=7.3 \mathrm{~Hz}$, $\mathrm{ArH}) ;{ }^{13} \mathrm{C} \mathrm{NMR}\left(\mathrm{CDCl}_{3}\right) \delta: 38.1,39.7,44.5,55.3,66.4,126.9,127.4,127.8,127.9,128.5,128.7,128.8,129.0$, $129.4,129.5,130.2,133.1,134.8,135.2,137.5,139.7,142.5,144.1,153.2,173.1 ;$ HRMS calcd for $\mathrm{C}_{33} \mathrm{H}_{29} \mathrm{NO}_{5} \mathrm{~S}$ $\left(\mathrm{M}^{+}\right)$551.1766, found 551.1760. 

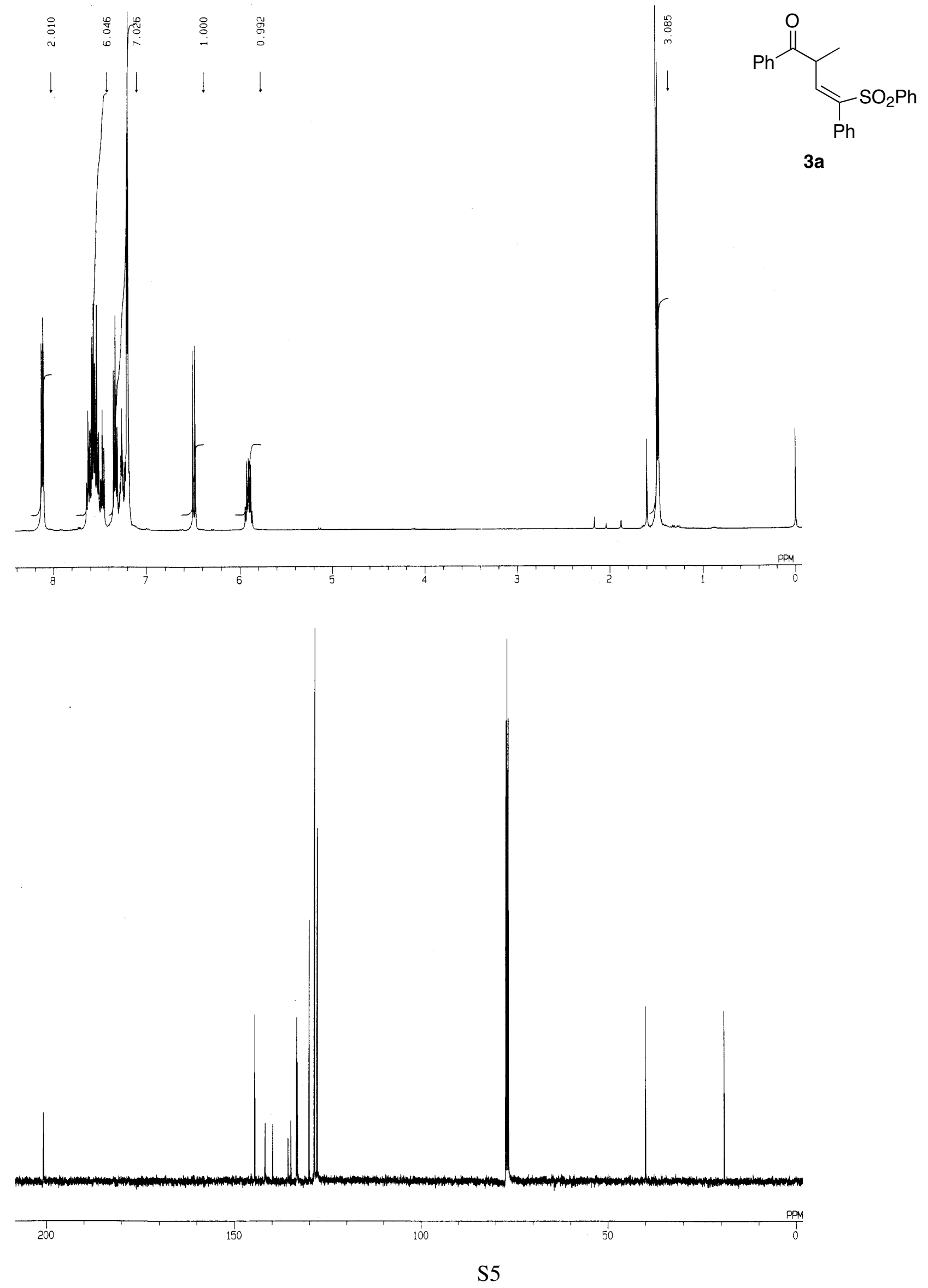

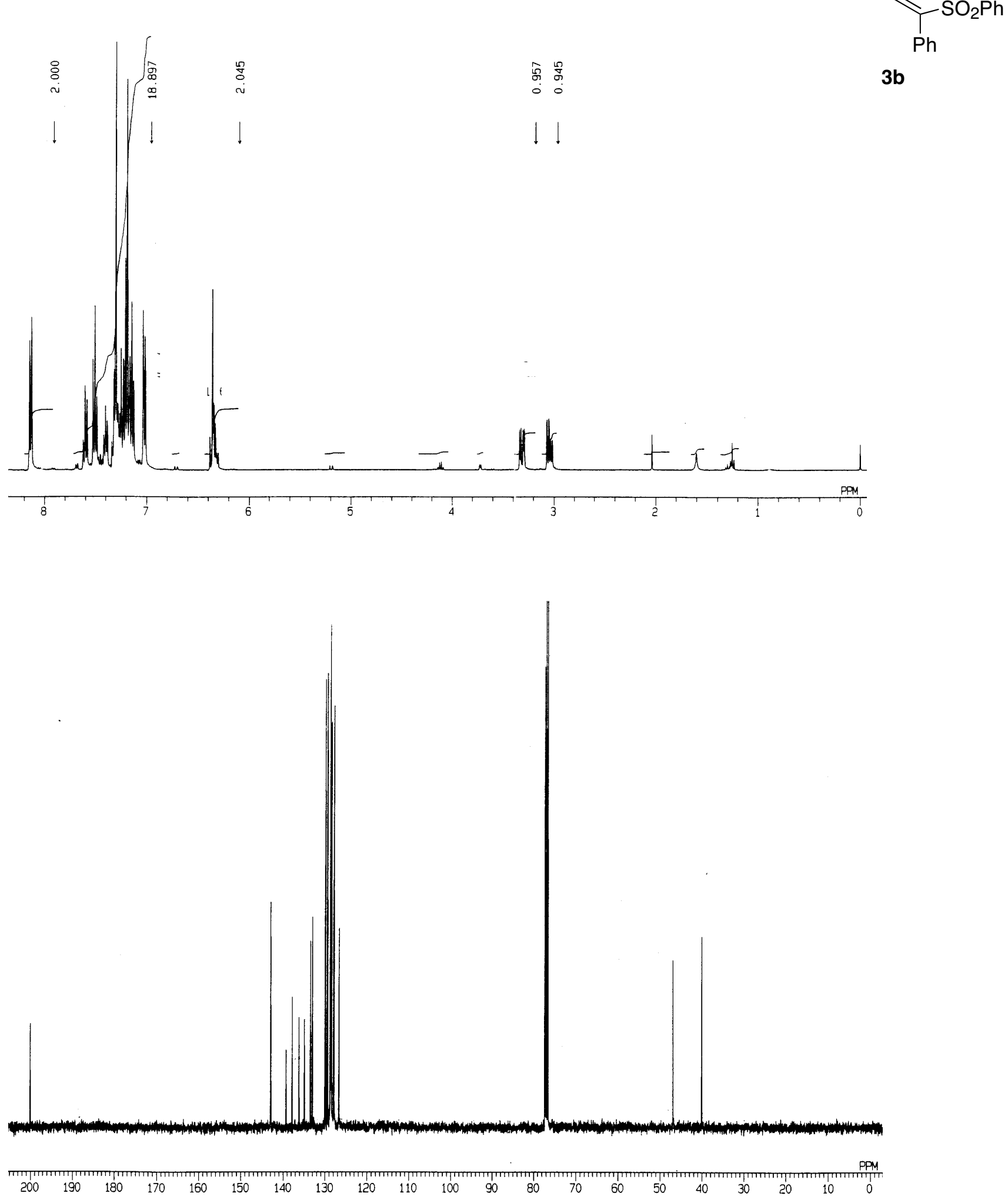

S6 

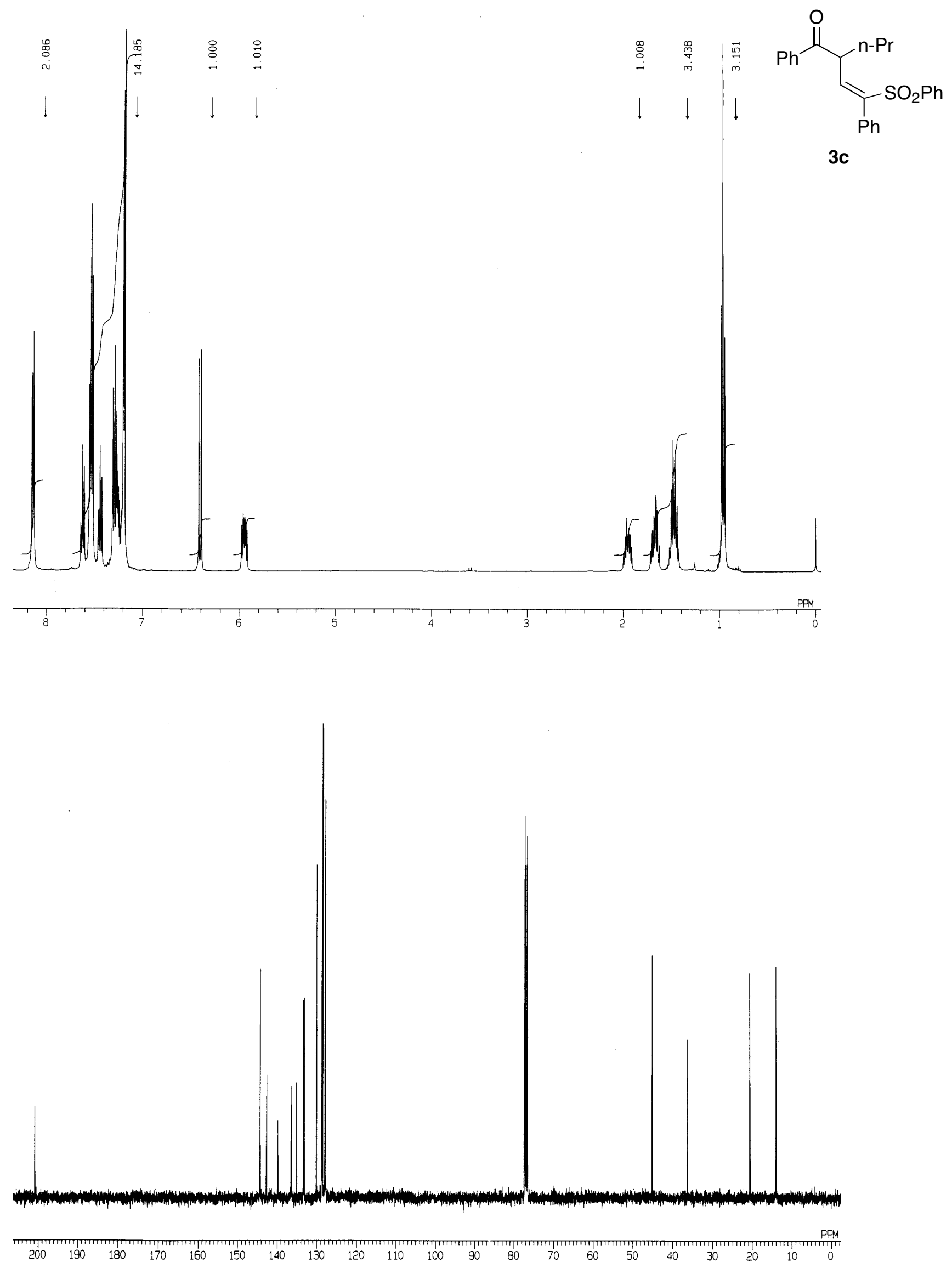


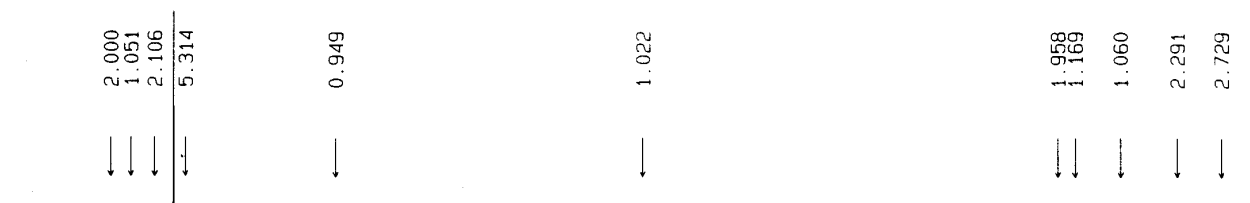<smiles>O=C1CCCCC1C=C(c1ccccc1)[SH](=O)(Oc1ccccc1)c1ccccc1</smiles>

3d
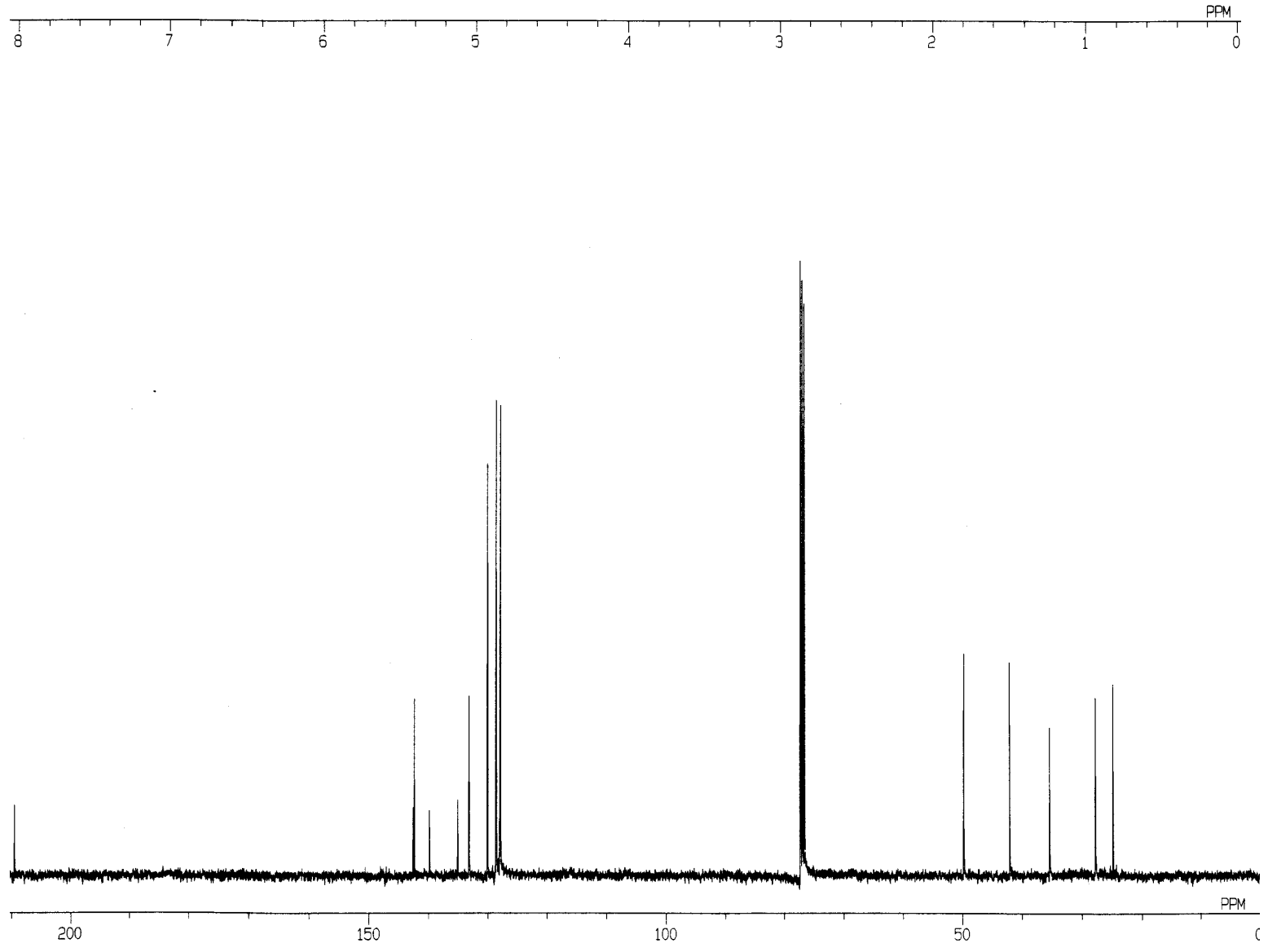

S8 

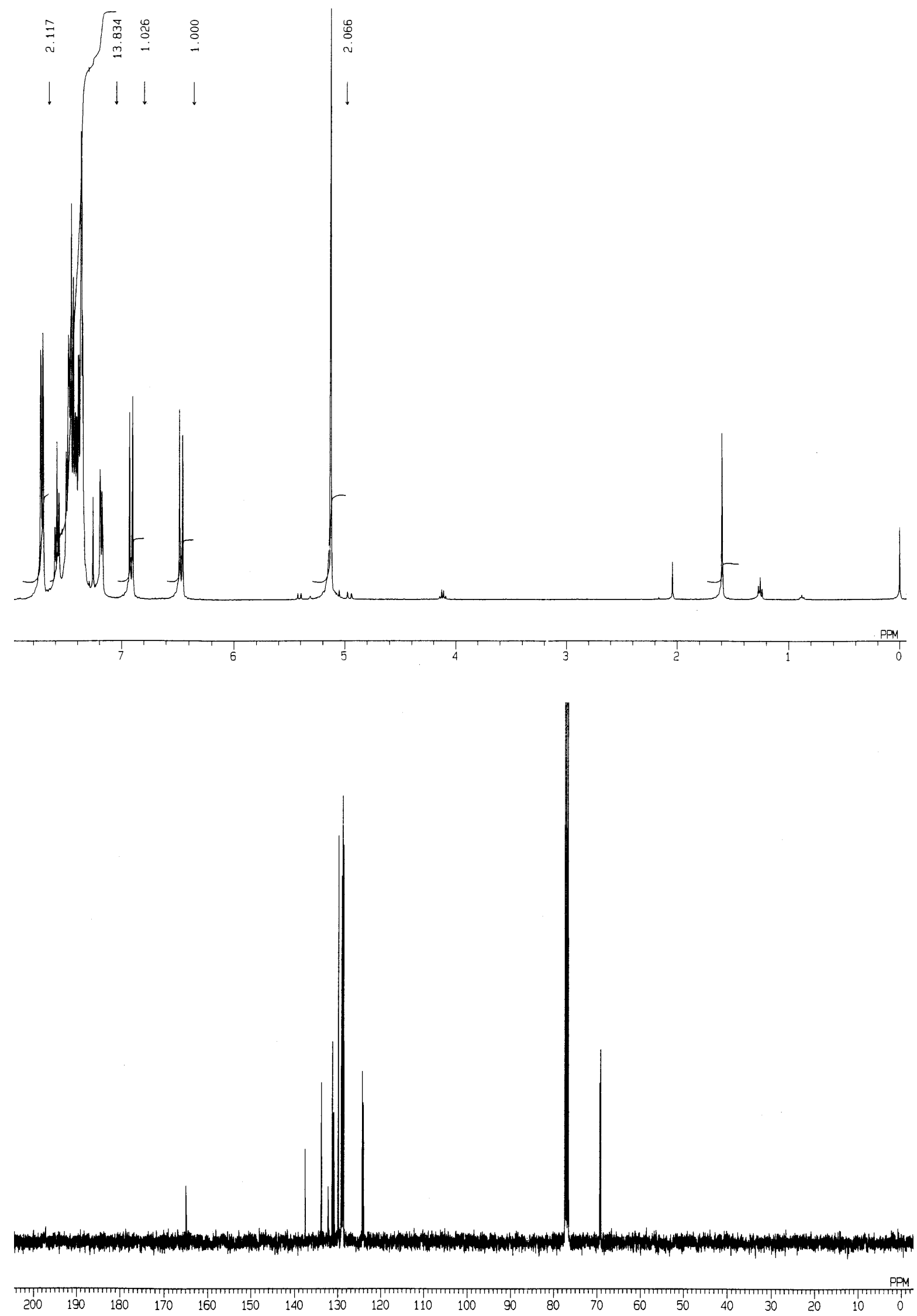

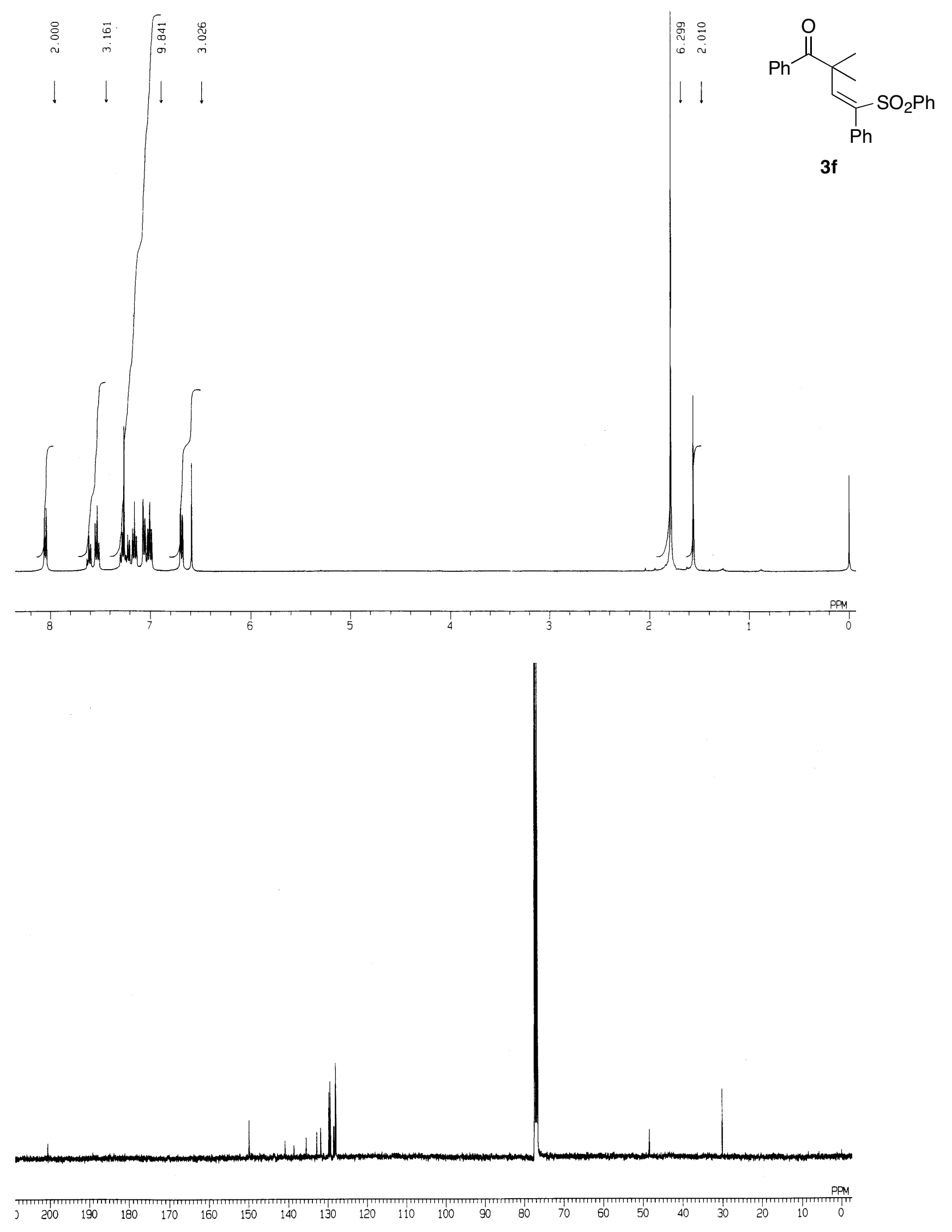

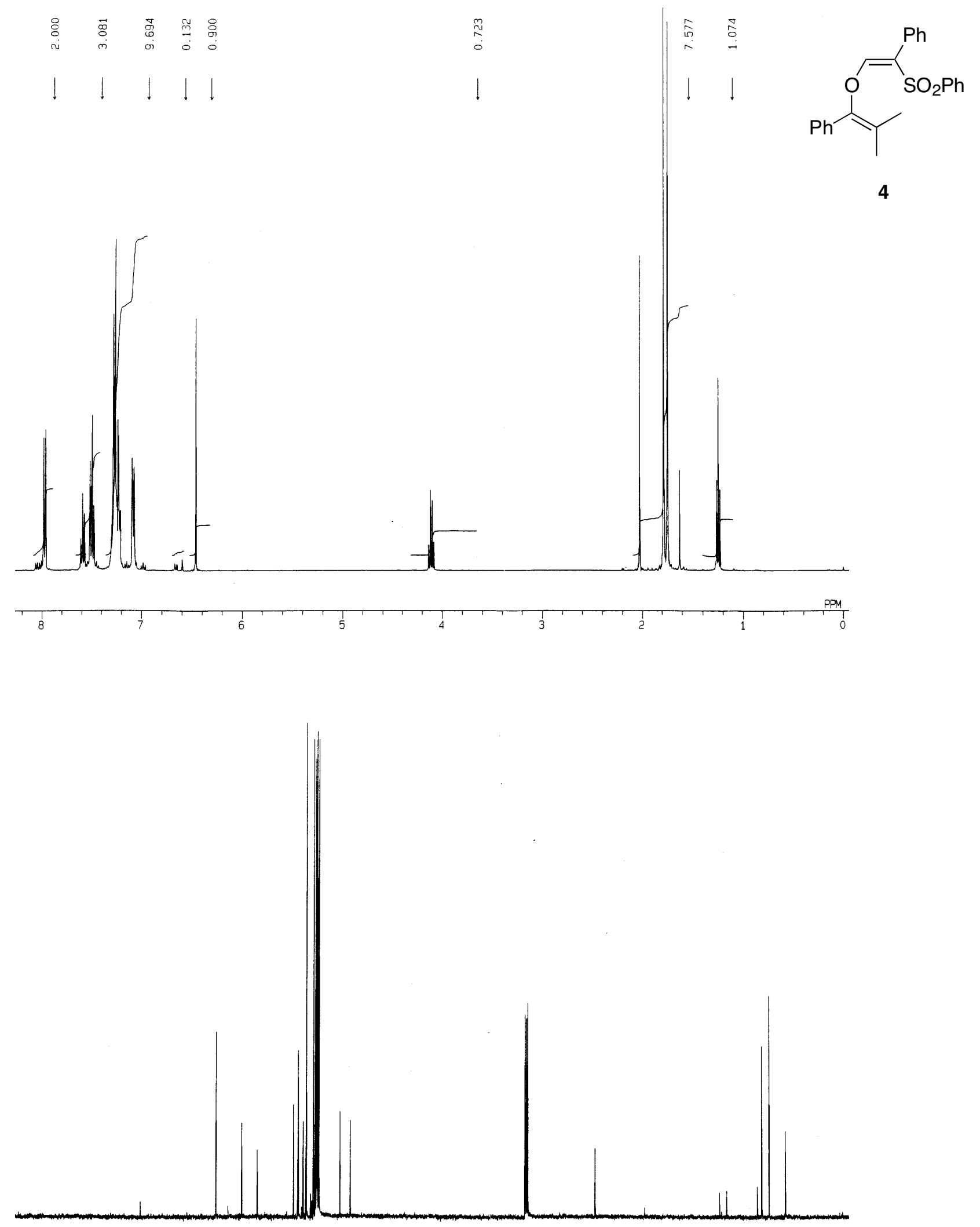

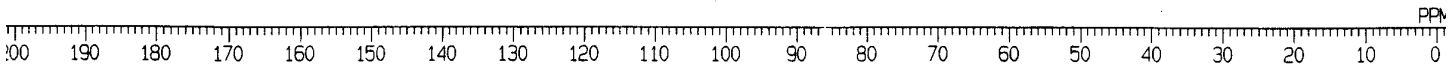




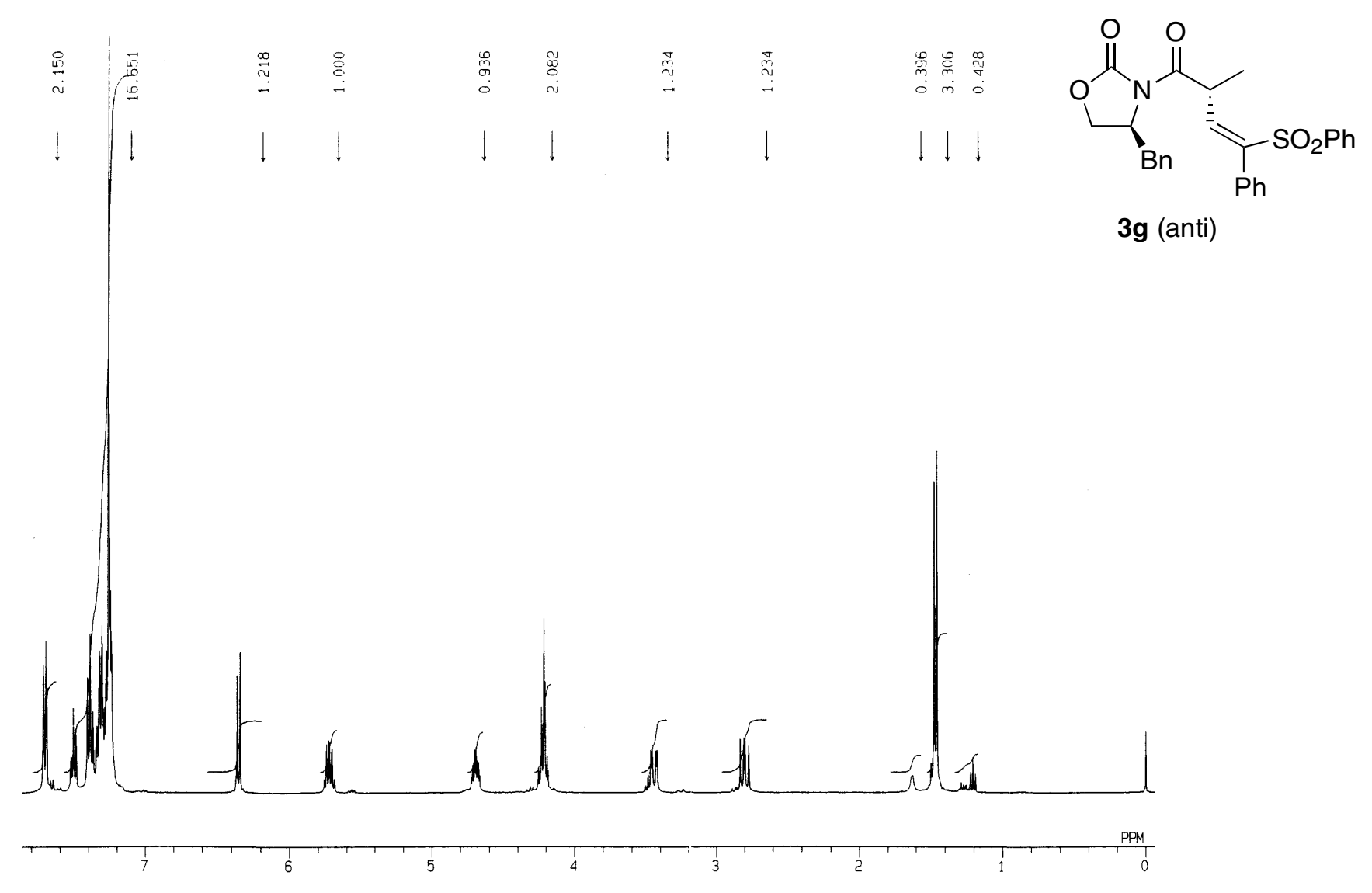



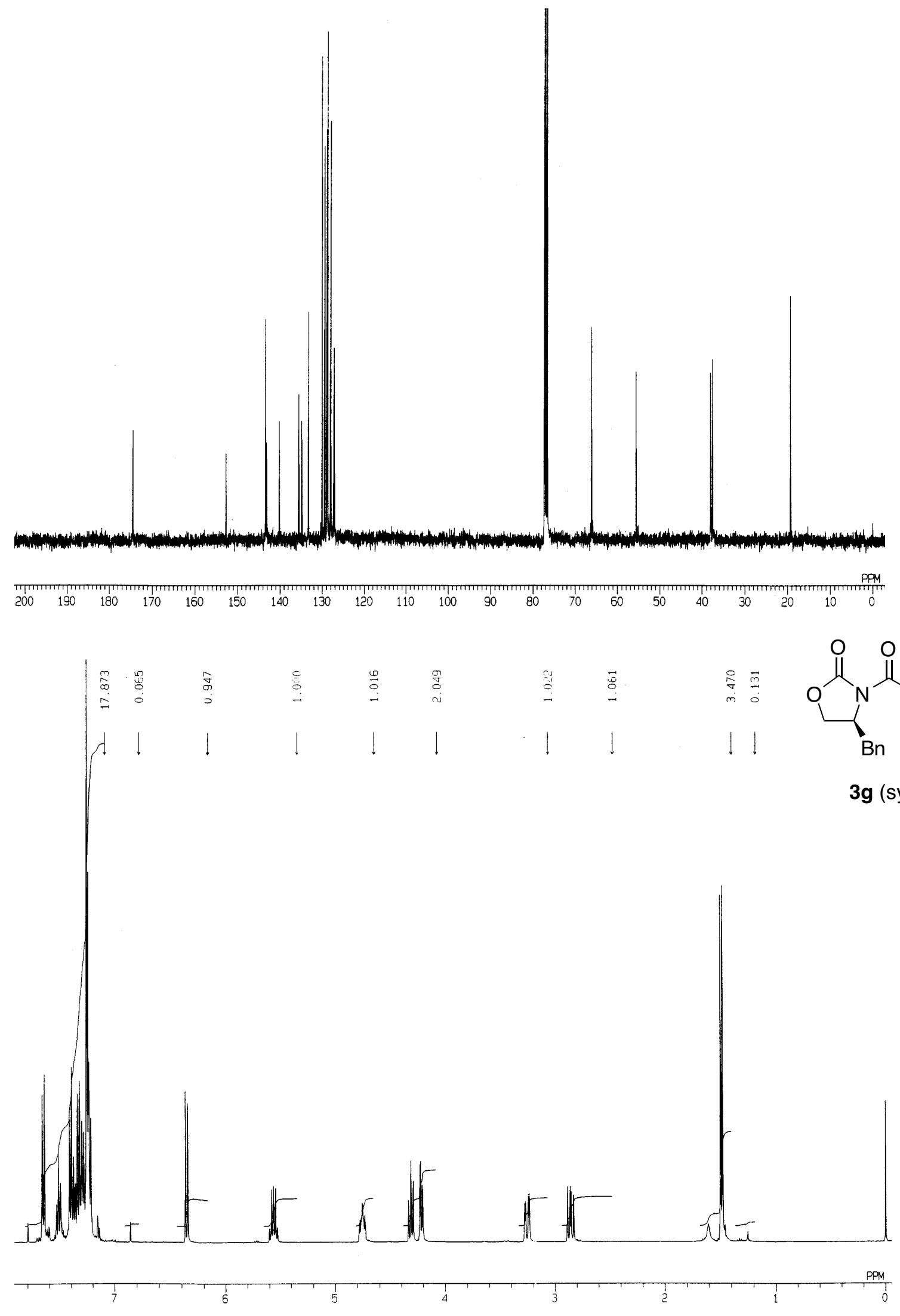


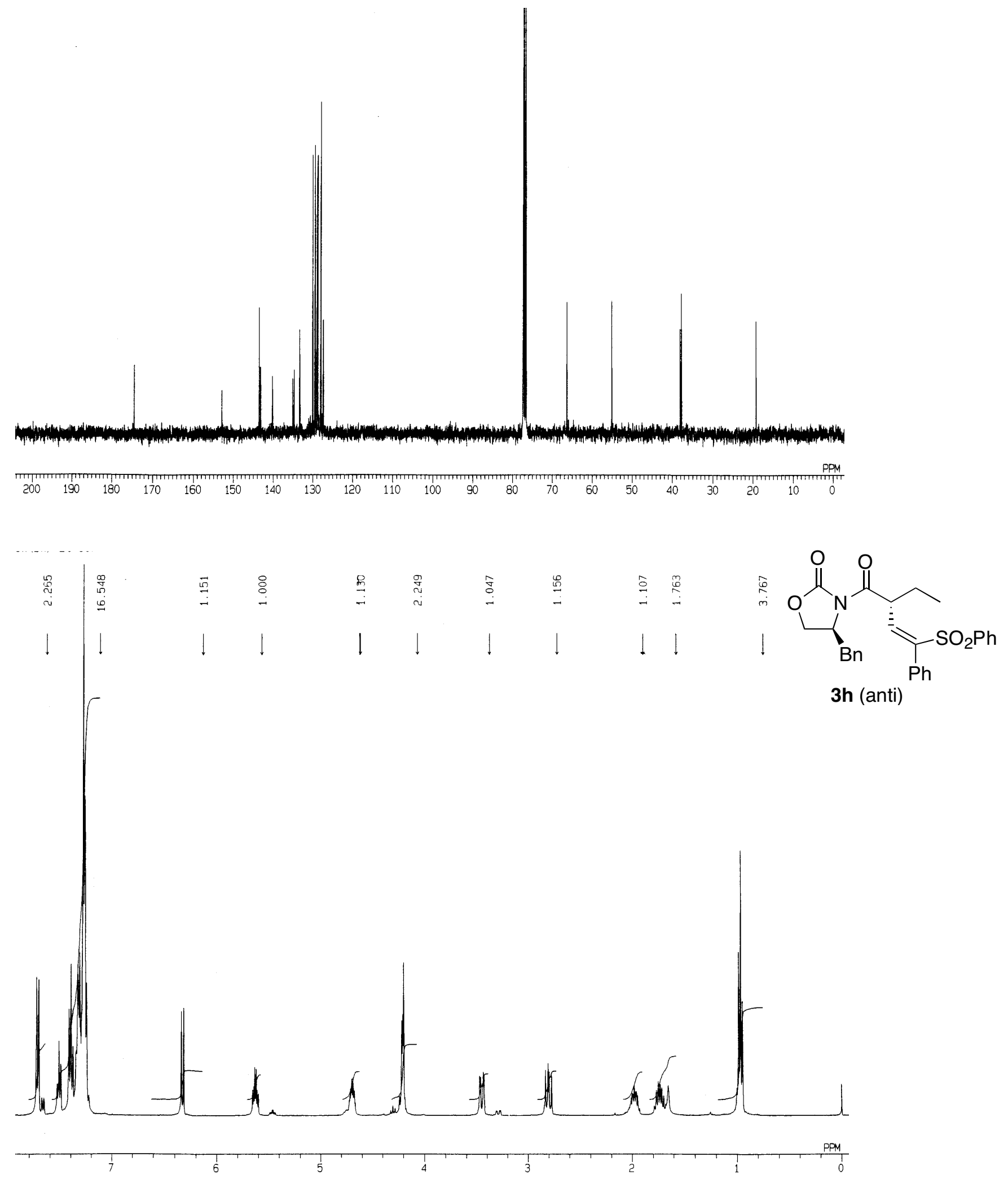




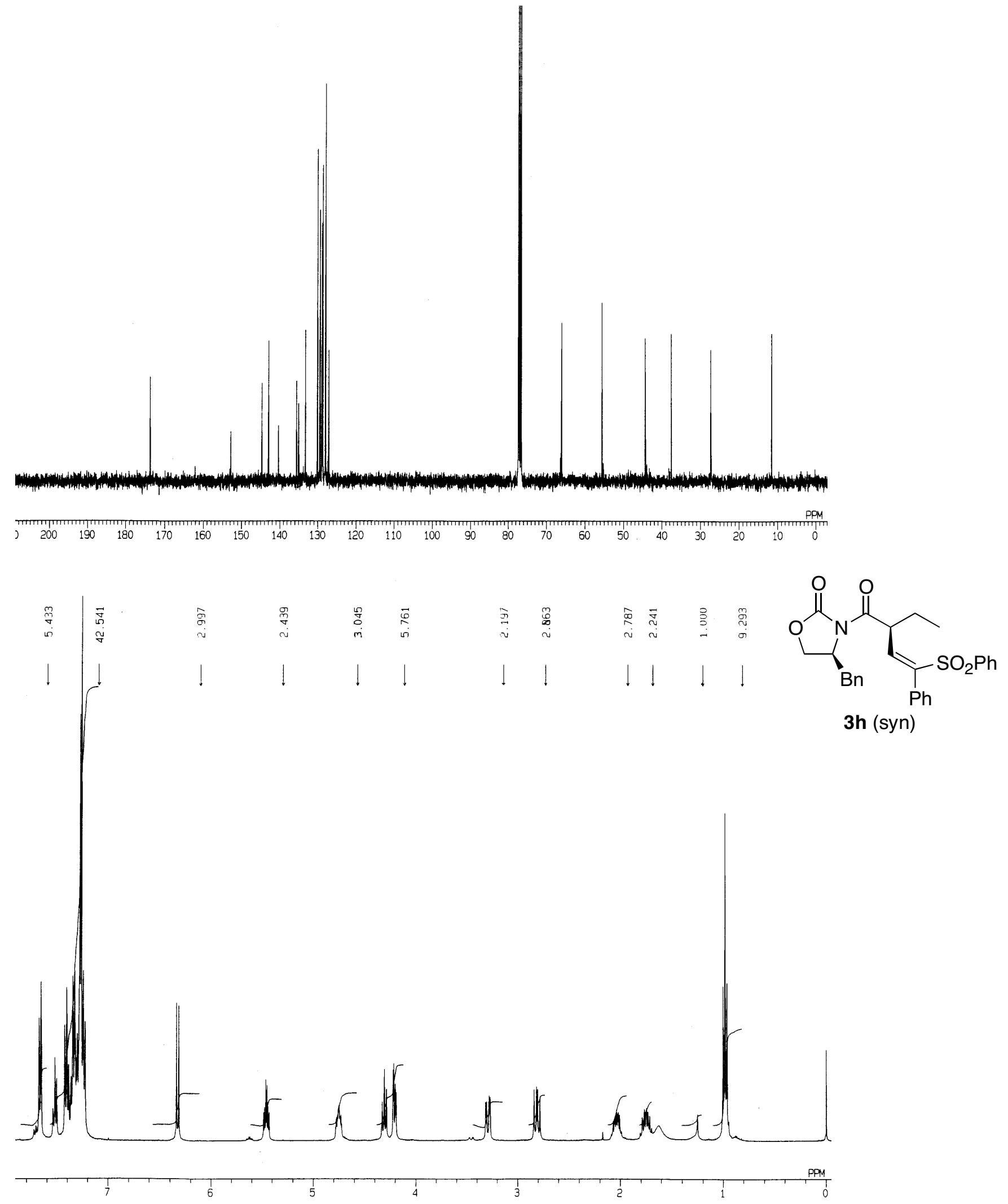




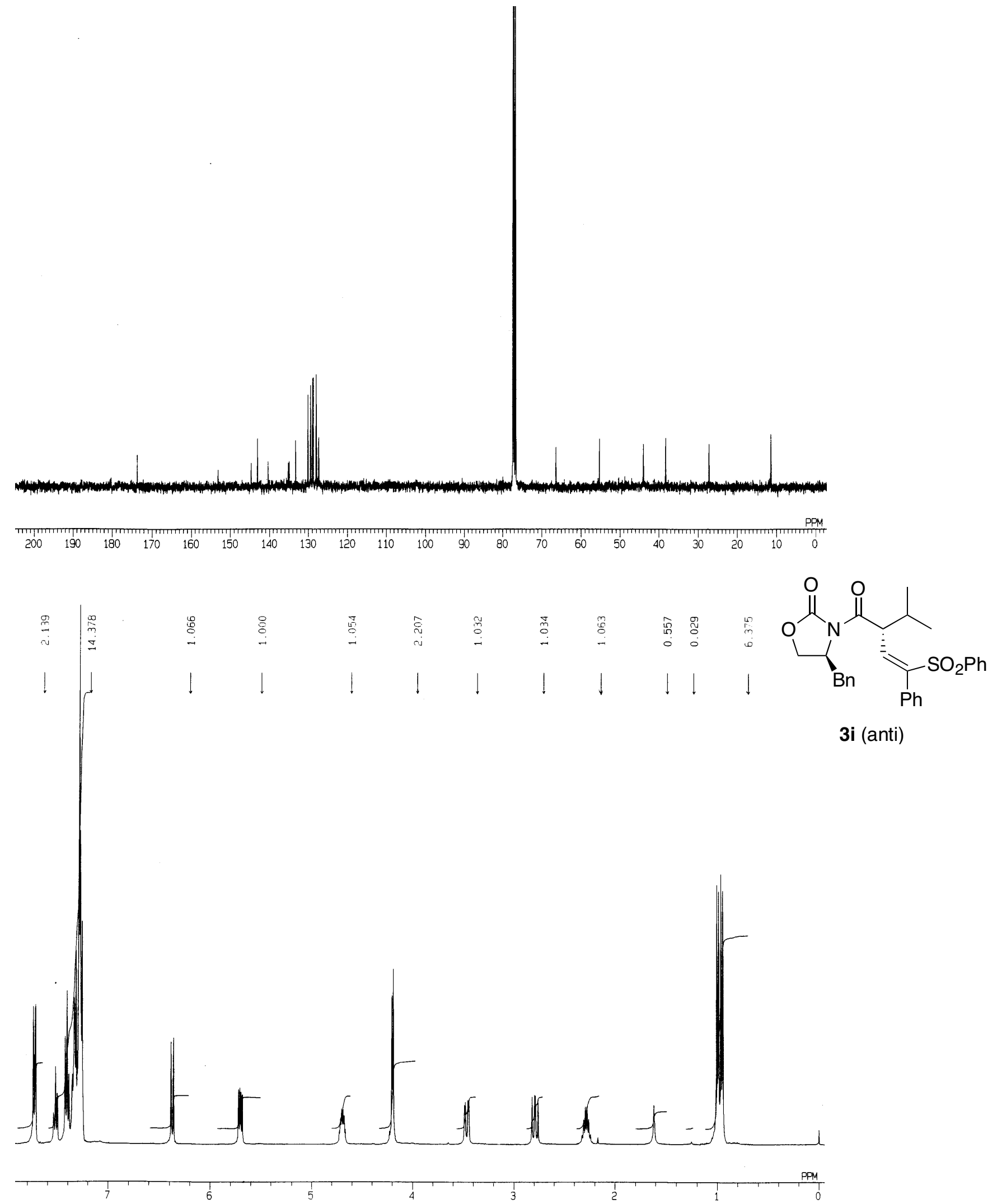



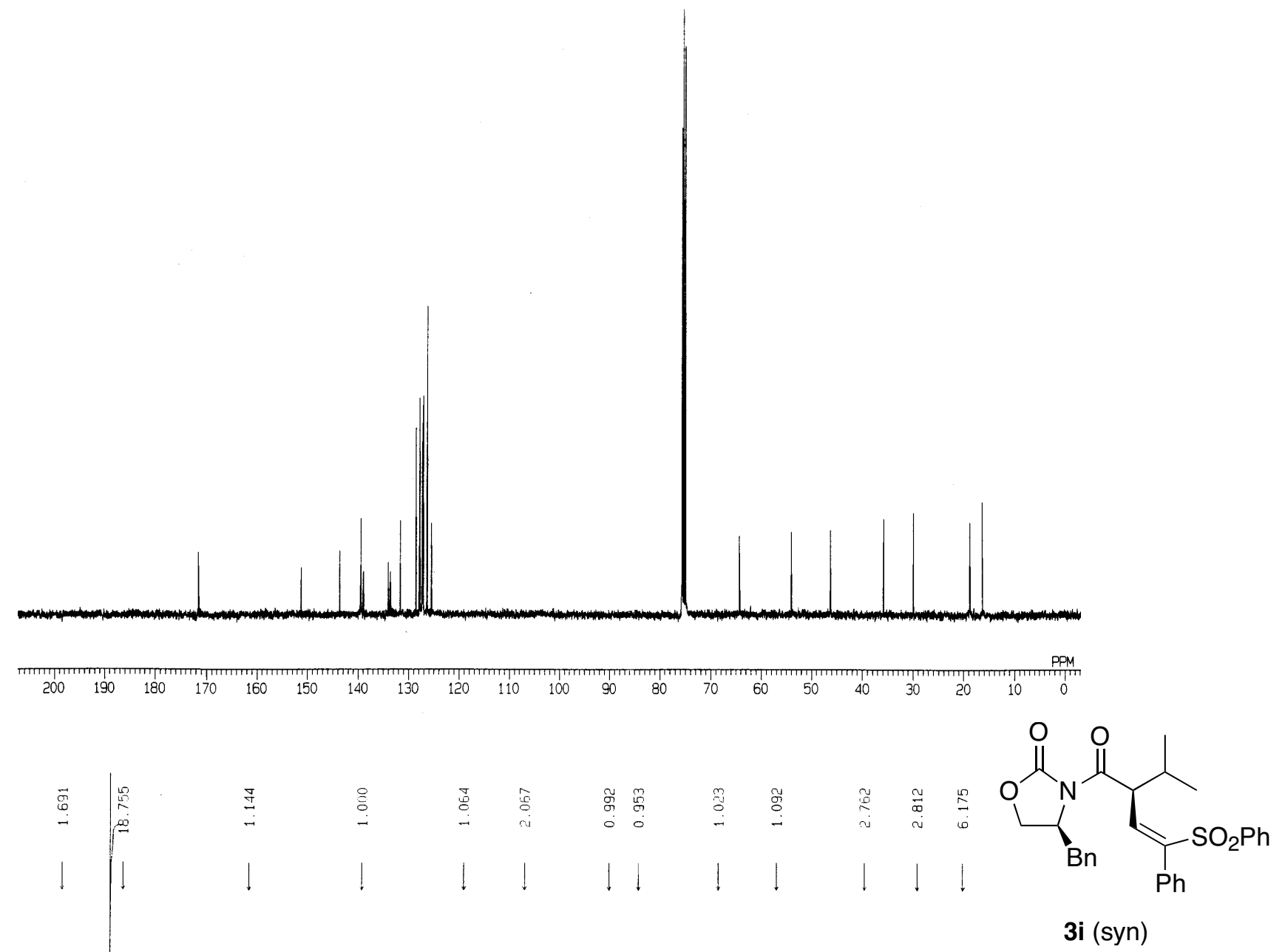

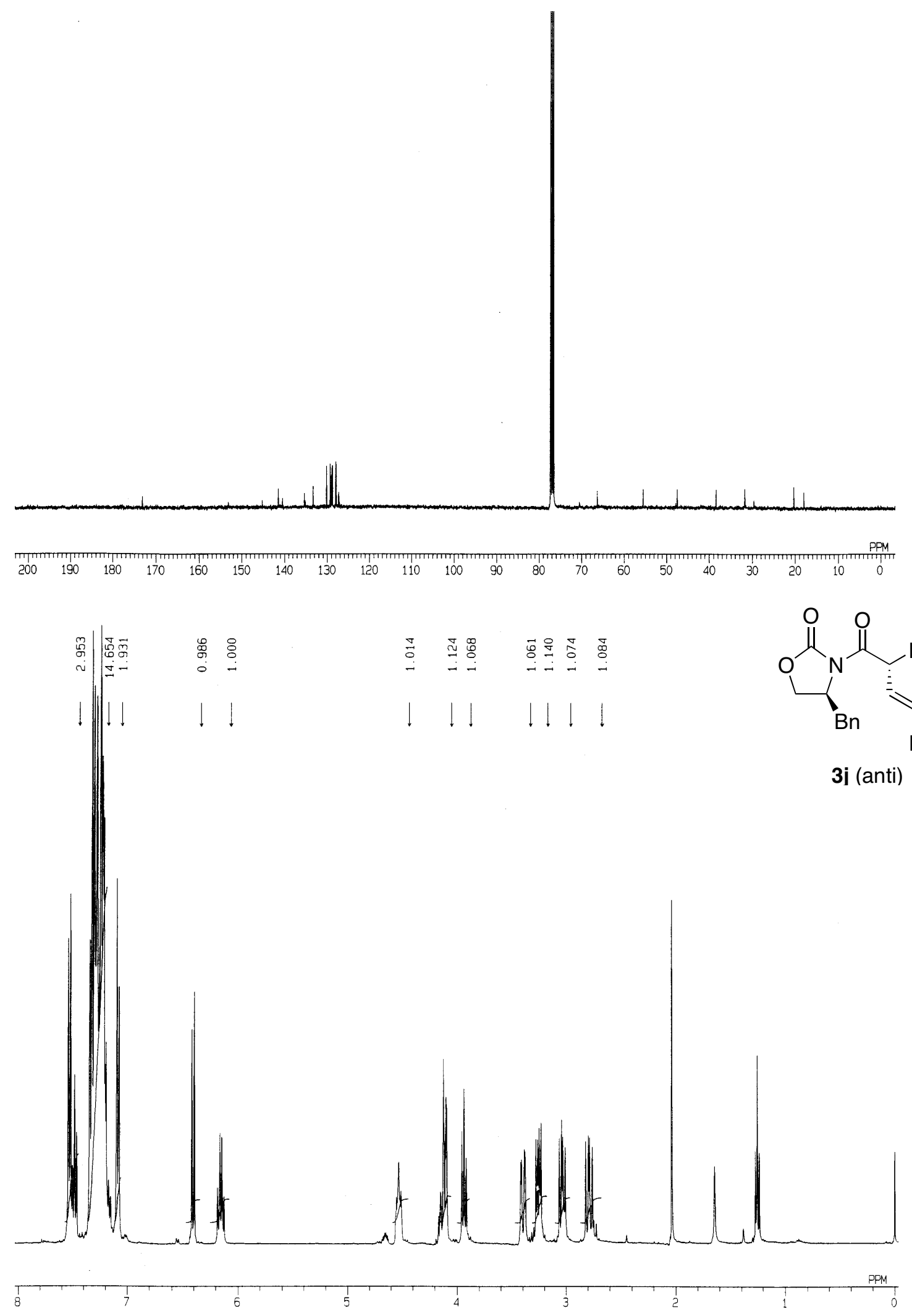

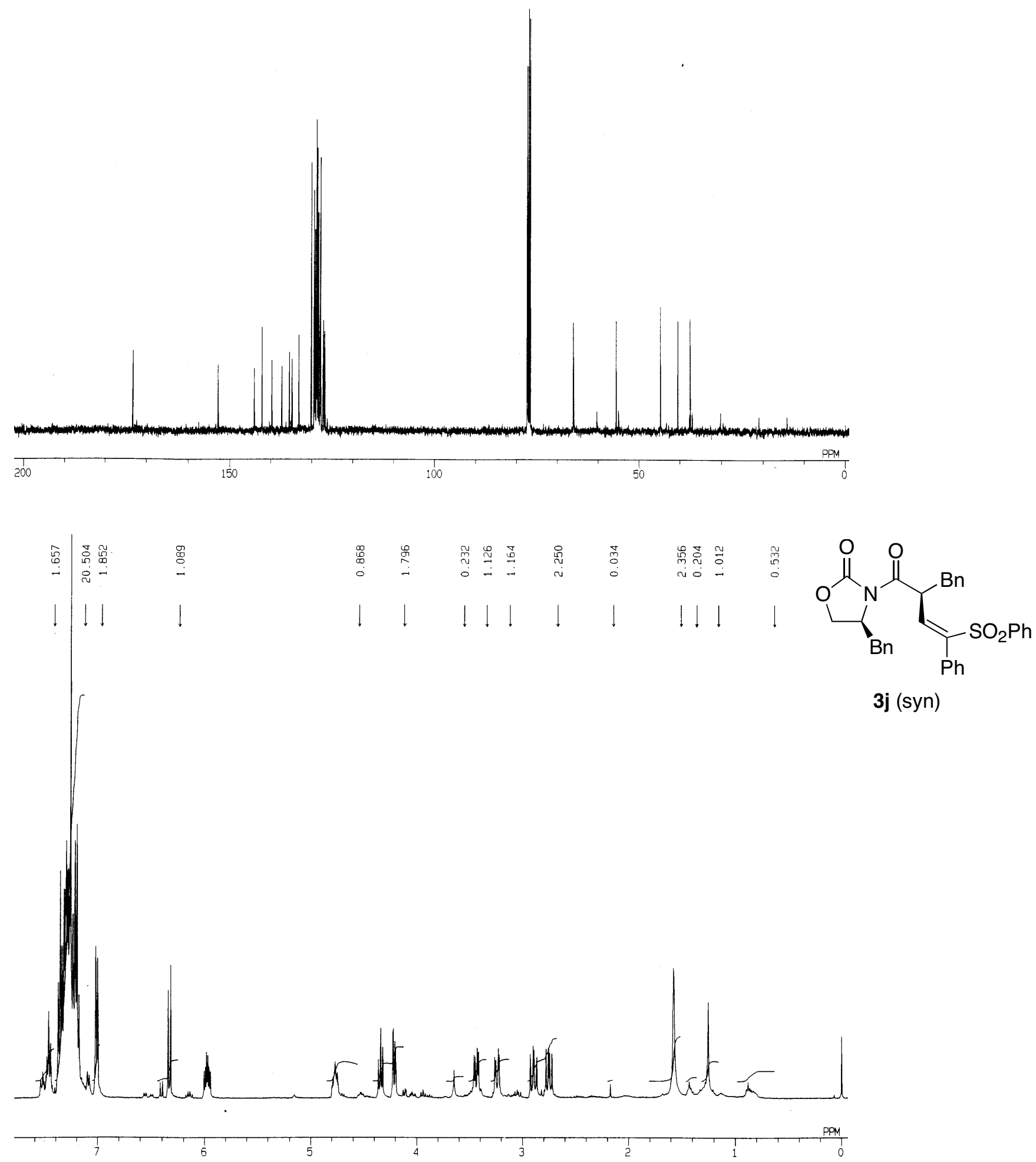


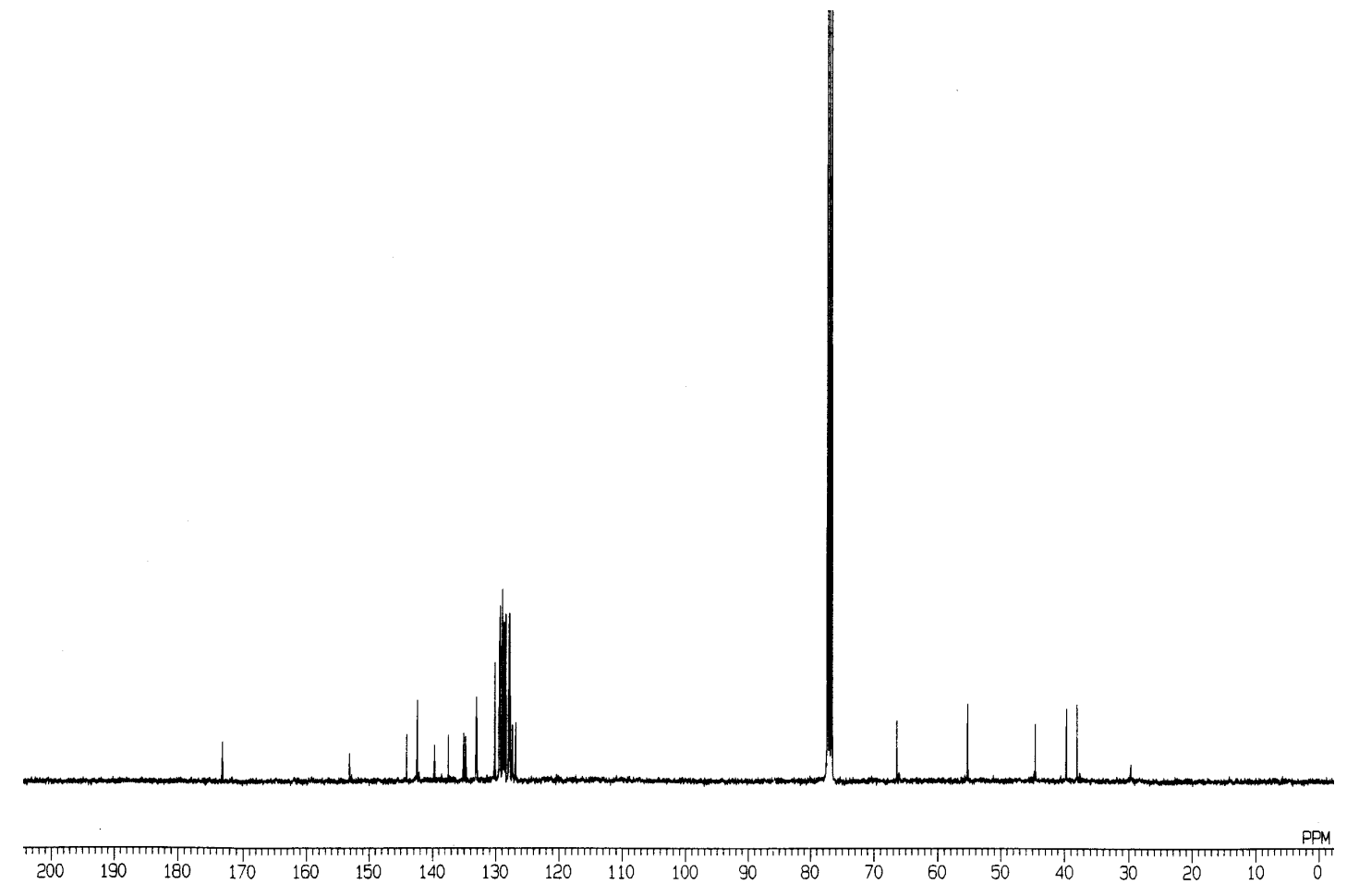

